\title{
Potentiometrischer Sensor für reduzierende Gase auf Basis von Zeolithen
}

\author{
Isabella Marr, Gunter Hagen und Ralf Moos
}

Lehrstuhl für Funktionsmaterialien, Universität Bayreuth, 95440 Bayreuth

\begin{abstract}
Die Materialklasse der Zeolithe findet seit einiger Zeit auch im Bereich der Gassensorik Anwendung. In dieser Arbeit wurden planare potentiometrische Gassensoren zur Detektion von Wasserstoff und Kohlenwasserstoffen ohne Reinraumprozesse in Dickschichttechnologie hergestellt. Zusätzlich wurde der Einfluss von Messtemperatur, Testgas, Dicke und Pt-Gehalt der Zeolithschicht und Dicke der sensitiven $\mathrm{Cr}_{2} \mathrm{O}_{3^{-}}$ Schicht, sowie die Ansprechzeit untersucht.
\end{abstract}

\section{Einleitung}

Im Bereich der Gassensorik werden Zeolithe zum einen als Filterschicht, zum anderen als sensitive Sensorschicht, mit sich ändernden elektrischen Eigenschaften, eingesetzt.[1,2] Zeolithe sind mikroporöse Alumosilikate und zeichnen sich durch ihre strukturbedingten, einzigartigen Eigenschaften aus. Durch ihre verzweigte Kanalstruktur und die damit verbundene hohe innere Oberfläche sind Zeolithe als Filtermaterial und katalytisch aktive Schicht geeignet. Zusätzlich besitzen sie in ihren Kanälen bewegliche austauschbare Kationen, was in einer ionischen Leitfähigkeit resultiert. Diese Kationen können durch Edelmetalle, wie beispielsweise Pt, Pd oder Ni, ausgetauscht werden, was zu einer weiteren katalytischen Aktivität führt [3].

In Ref. [4] und [5] wurde der Einfluss einer $\mathrm{Cr}_{2} \mathrm{O}_{3}$-Schicht zwischen Goldelektrode und funktioneller Zeolithschicht bei Beaufschlagung von impedimetrischen Sensoren mit $\mathrm{C}_{3} \mathrm{H}_{8}$ untersucht. Fehlt diese Zwischenschicht, so zeigte der Sensor keine Signaländerung in einer kohlenwasserstoffhaltigen Gasatmosphäre. Ist die $\mathrm{Cr}_{2} \mathrm{O}_{3}$-Schicht hingegen vorhanden, ist eine deutliche Signaländerung zu messen.

Die Eigenschaften der Zeolithe sowie der Einfluss der $\mathrm{Cr}_{2} \mathrm{O}_{3}$-Schicht wurden nun genutzt, um einen selektiven potentiometrischen Sensor für reduzierende Gase herzustellen.

\section{Experimentelles}

Zur Herstellung der Sensoren wurde zunächst eine interdigitale Goldelektrodenstruktur (Fingerbreite und Fingerabstand $100 \mu \mathrm{m}$ ) mittels Siebdrucktechnik (Goldpaste Heraeus KQ 500) auf $\mathrm{Al}_{2} \mathrm{O}_{3}$-Substrate realisiert. Anschließend wurde jeweils eine Elektrode galvanisch mit einer Cr-Beschichtung versehen [6].

Die austauschbaren $\mathrm{Na}^{+}$-Ionen wurden durch Ionenteilaustausch durch Platin ersetzt, was die katalytische Aktivität des eingesetzten Zeoliths Na-ZSM-5 (Süd-Chemie AG) zusätzlich erhöht. Die Beladung des Zeolithen mit 1, 2 oder 3 Gew.-\% Pt erfolgte in wässriger Lösung mittels Tetraamminplatin(II)chlorid (56 Gew.-\% Pt). Die anschließende Reduktion wurde in wässriger $\mathrm{NaBH}_{4}$-Lösung bei Raumtemperatur oder im $\mathrm{H}_{2} / \mathrm{N}_{2}$-Gasstrom bei einer Temperatur von $450{ }^{\circ} \mathrm{C}$ durchgeführt [7]. Die hergestellten Pulver wurden mit Hilfe organischer Binder zu einer siebdruckfähigen Paste verarbeitet.

Die Zeolithpasten wurden mittels Siebdruckverfahren auf die einseitig verchromten Au-Interdigitalelektroden aufgebracht und bei $450{ }^{\circ} \mathrm{C}$ gebrannt. Durch das Einbrennen wurde das organische Bindemittel aus der Paste entfernt, wie auch die Cr-Schicht $\mathrm{zu} \mathrm{Cr}_{2} \mathrm{O}_{3}$ oxidiert. Das entstandene Oxid bildet die sensitive Grenzfläche zwischen Pt-beladenem Zeolith und Au-Elektrode. In Bild 1 ist der Aufbau der planaren Zeolithsensoren schematisch in Draufsicht und im Querschnitt gezeigt. 

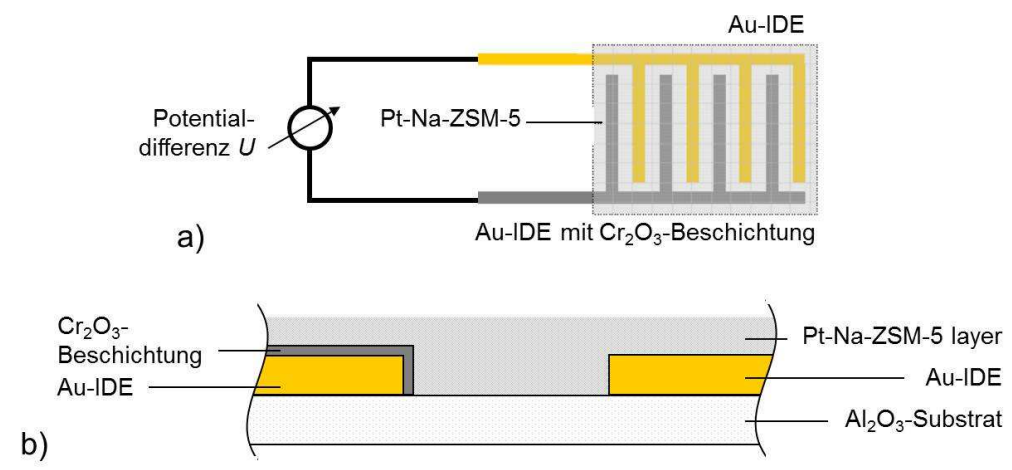

Bild 1 Schematischer Aufbau des potentiometrischen Zeolithsensors (a) in Draufsicht und (b) im Querschnitt

Zur Untersuchung des Einflusses der verschiedenen Parameter (Testgas, Messtemperatur, Pt-Gehalt der Zeolithschicht, Dicke der Zeolithschicht, Dicke der Cr-Beschichtung) wurden die Sensoren in einen Rohrofen eingebaut, aufgeheizt und mit Testgasen beaufschlagt. Der Grundgasstrom setzte sich dabei immer aus $10 \% \mathrm{O}_{2}$ und 2,5\% $\mathrm{H}_{2} \mathrm{O}$ in $\mathrm{N}_{2}$ zusammen. Der Gasfluss durch den Ofen wurde auf $600 \mathrm{ml} / \mathrm{min}$ eingestellt. Als Testgase wurden $\mathrm{H}_{2}, \mathrm{CH}_{4}, \mathrm{C}_{2} \mathrm{H}_{6}, \mathrm{C}_{3} \mathrm{H}_{8}$ und $\mathrm{n}-\mathrm{C}_{4} \mathrm{H}_{10}$, mit einer Konzentration von jeweils $500 \mathrm{ppm}$, verwendet. Als Sensorsignal dient die Spannungsdifferenz $\Delta U$, die sich aus der Differenz der gemessenen Spannung des Sensors im Grundgas und der gemessenen Spannung bei Beaufschlagung mit Testgas ergibt.

\section{Messergebnisse und Diskussion}

\subsection{Einfluss der Chrombeschichtung}

Zur Untersuchung des Einflusses der $\mathrm{Cr}_{2} \mathrm{O}_{3}$-Beschichtung auf einer der Elektroden auf das Sensorsignal wurden Sensoren mit unterschiedlicher Cr-Schichtdicke hergestellt. Die Schichtdicken betrugen 75, 150 und $251 \mathrm{~nm}$. Die Sensoren wurden mit einer Zeolithschicht mit einem Pt-Gehalt von 3 Gew.-\% versehen. Die Messungen wurden bei 300,350 und $400{ }^{\circ} \mathrm{C}$ mit $500 \mathrm{ppm}_{2}$ als Testgas durchgeführt.

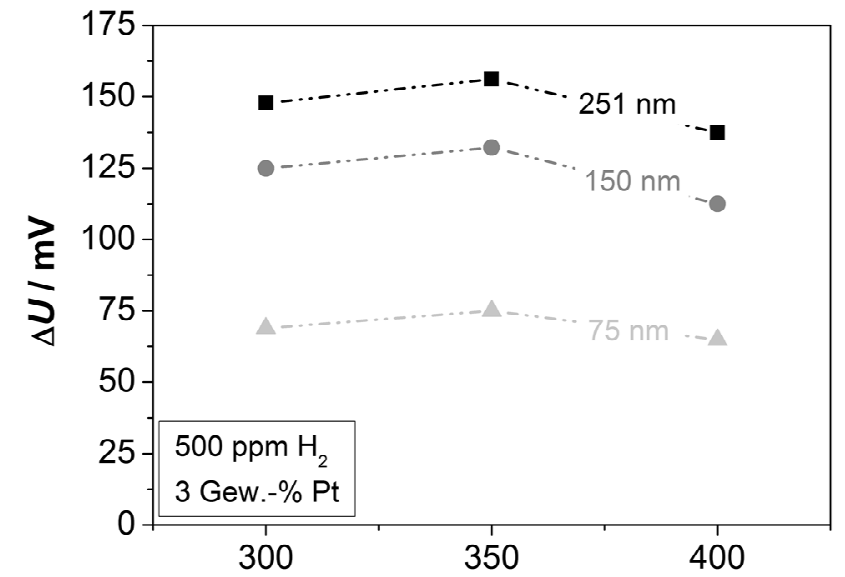

a)

$T /{ }^{\circ} \mathrm{C}$

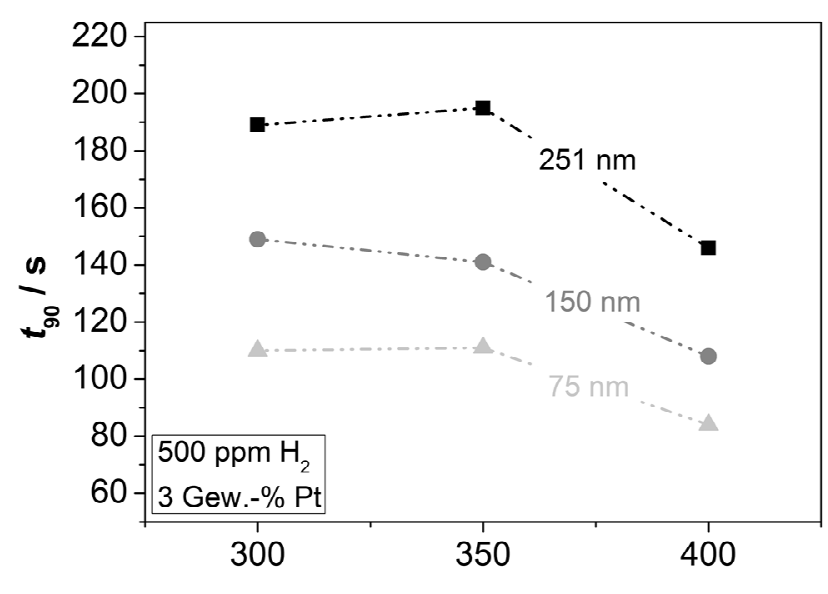

b)

Bild 2 (a) Sensorsignal und (b) Ansprechzeit der Sensoren mit unterschiedlich dicker $\mathrm{Cr}_{2} \mathrm{O}_{3}$-Schicht aufgetragen über die Messtemperatur

In Bild 2 sind (a) die Signaländerung $\Delta U$ und (b) die Ansprechzeit $t_{90}$ der Sensoren in Abhägigkeit der Messtemperatur dargestellt. Deutlich zu erkennen ist, dass mit steigender $\mathrm{Cr}_{2} \mathrm{O}_{3}$-Schichtdicke auch das Sensorsignal zunimmt. Dies lässt auf eine Erhöhung der Empfindlichkeit des Sensors durch die $\mathrm{Cr}_{2} \mathrm{O}_{3^{-}}$ Beschichtung auf einer Elektrode schließen. Allerdings nimmt die Ansprechzeit ebenfalls mit steigernder $\mathrm{Cr}_{2} \mathrm{O}_{3}$-Schichtdicke zu. Die Messtemperatur hat nur einen geringen Einfluss auf das Sensorsignal. Lediglich die Ansprechzeit nimmt bei $400{ }^{\circ} \mathrm{C}$ im Vergleich zu den Ansprechzeiten bei 300 und $350{ }^{\circ} \mathrm{C}$ ab. 


\subsection{Einfluss des Testgases}

Um zu untersuchen, welchen Einfluss die verschiedenen Testgase auf das Sensoresignal haben, wurden Sensoren mit gleicher $\mathrm{Cr}_{2} \mathrm{O}_{3}$-Beschichtung auf einer der interdigitalen Elektroden hergestellt und mit einer Zeolithschicht bedruckt. Der Pt-Gehalt des Zeoliths betrug 1, 2 oder 3 Gew.-\%.

In Bild 3 (a) ist das Sensorsignal bei Beaufschlagung mit Kohlenwasserstoffen (Konzentration jeweils 500 ppm) bei $300{ }^{\circ} \mathrm{C}$ über die Kettenlänge des Alkans aufgetragen. Bild 3 (b) zeigt die Spannungsdifferenz bei Zudosierung von Wasserstoff in Abhängigkeit von der Temperatur. Ein deutlicher Einfluss des Testgases ist, dass die Sensoren bei Zudosierung von Alkanen mit einer Spannungsabnahme, bei Zudosierung von Wasserstoff jedoch mit einer Spannungszunahme reagieren. Des Weiteren ist die Sensorantwort auf $\mathrm{H}_{2}$ deutlich höher als auf Kohlenwasserstoffe, was durch die schnellere Diffusion der $\mathrm{H}_{2}$-Moleküle und der höheren Reaktivität, verglichen mit den Alkanen, erklärt werden kann.

Betrachtet man sich die in Bild 3 (a) aufgetragenen Spannungsdifferenzen, so ist eine deutliche Abhängigkeit des Sensorsignals von der Kettenlänge des zudosierten Alkans zu beobachten. Mit steigender Kettenlänge nimmt auch die Sensorantwort zu. Auch der Pt-Gehalt der Zeolithschicht beeinflusst das Sensorsignal; mit steigendem Pt-Gehalt nimmt das Signal ab. Die in Bild 3 (b) gezeigten Sensorantworten auf $\mathrm{H}_{2}$ weisen keine bestimmte Abhängigkeit von Temperatur und Pt-Gehalt auf. Die Sensoren mit einem Pt-Gehalt von 2 Gew.\% zeigen unabhängig von der Messtemperatur die stärksten Spannungsänderungen. Unabhängig vom PtGehalt nimmt die Spannungsdifferenz für alle Sensoren bei $400{ }^{\circ} \mathrm{C}$ ab. Da Wasserstoff sehr reaktiv ist, reagiert vermutlich ein großer Teil des $\mathrm{H}_{2}$ bei $400{ }^{\circ} \mathrm{C}$ schon bei der Diffusion durch den Zeolithen, was in einer verringerten Sensorantwort resultiert.

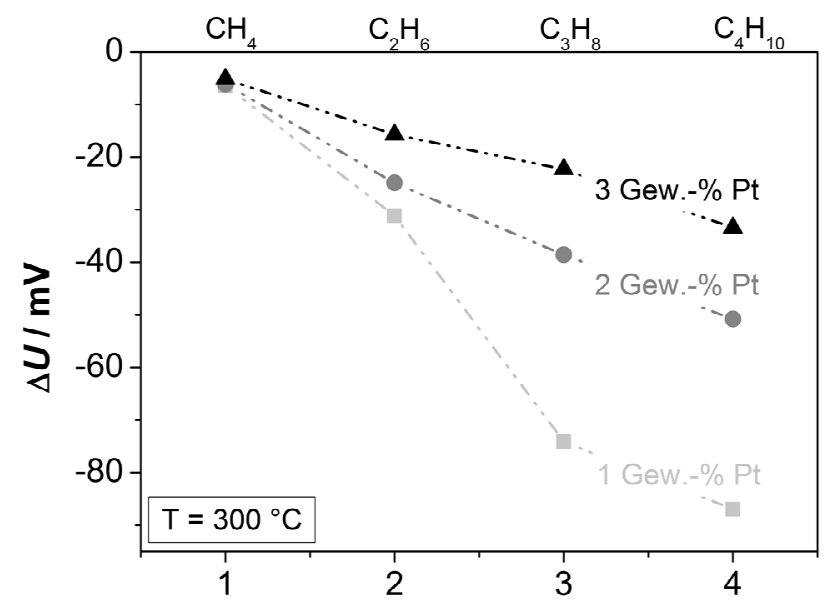

a)

Kettenlänge des Alkans

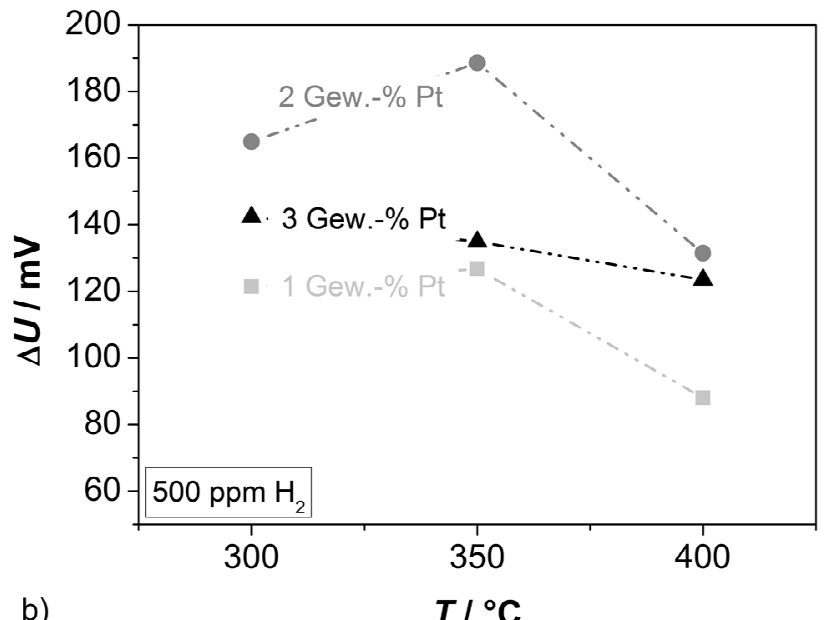

Bild 3 Sensorantwort (a) bei $300^{\circ} \mathrm{C}$ aufgetragen über die Kettenlänge der zudosierten Alkane und (b) auf $\mathrm{H}_{2}$ aufgetragen über die Temperatur

\subsection{Einfluss der Dicke der Zeolithschicht}

Um den Einfluss der Zeolithschichtdicke zu betrachten, wurden Sensoren hergestellt, die ein, zwei, drei und vier Mal mit einer Zeolithpaste mit 2 Gew.-\% Pt bedruckt wurden. Pro Schicht beträgt die Dicke ungefähr $25 \mu \mathrm{m}$. Die Dicke der $\mathrm{Cr}_{2} \mathrm{O}_{3}$-Beschichtung war bei den hier untersuchten Sensoren jeweils im Bereich von $250 \mu \mathrm{m}$. Die Messung wurde bei $350{ }^{\circ} \mathrm{C}$ und mit verschiedenen Kohlenwasserstoffen mit einer Konzentration von jeweils 500 ppm durchgeführt.

In Bild 4 ist das Sensorsignal auf verschiedene Alkane in Abhängigkeit der Anzahl an Zeolithschichten aufgetragen. Bei einer Schichtdicke von $\sim 50 \mu \mathrm{m}$ (entspricht 2 Schichten) ist unabhängig vom zudosierten Alkan die größte Spannungsänderung $\mathrm{zu}$ beobachten. Dies könnte durch die Reaktion der Kohlenwasserstoffe während der Diffusion durch die Zeolithschicht erklärt werden. Besonders deutlich ist dies bei $\mathrm{CH}_{4} \mathrm{zu}$ erkennen, da bei mehr als zwei Zeolithschichten keine Spannungsänderung gemessen wurde. Allerdings wird auch hier wieder die Abhängigkeit des Sensorsignals von der Kettenlänge des Alkans 
deutlich. Die Spannungsdifferenz nimmt mit steigender Anzahl an Kohlenstoffatomen zu, allerdings ist der Unterschied zwischen $\mathrm{C}_{3} \mathrm{H}_{8}$ und $\mathrm{n}-\mathrm{C}_{4} \mathrm{H}_{10}$ nur noch sehr gering.

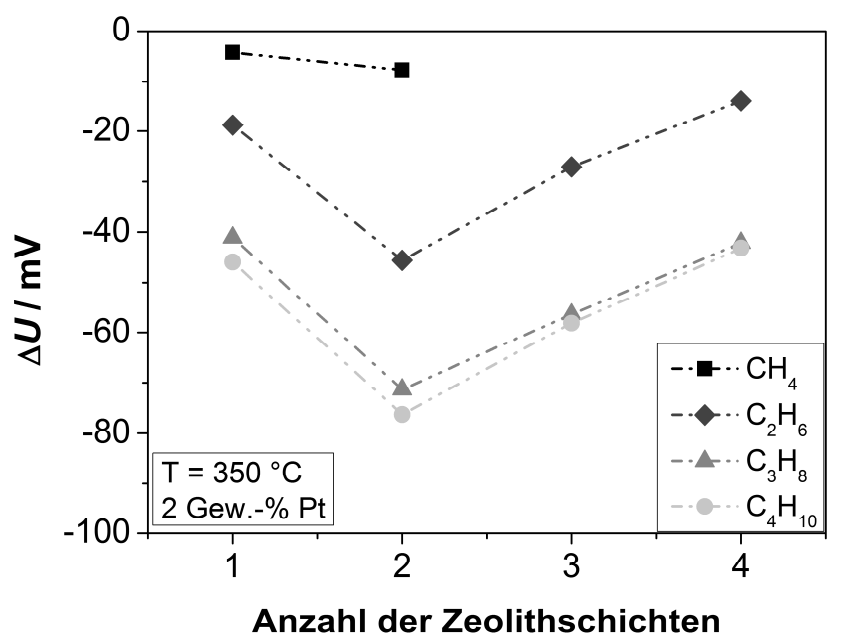

Bild 4 Sensorsignal der Sensoren mit unterschiedlicher Zeolithschichtdicke auf verschiedene Alkane bei $350{ }^{\circ} \mathrm{C}$

\section{Zusammenfassung}

In dieser Arbeit wurden erfolgreich selektive potentiometrische Gassensoren für reduzierende Gase auf Basis von Zeolithen hergestellt. Die Herstellung erfolgte mit kostengünstigen Techniken in Dickschichttechnologie und ohne Reinraumprozesse.

Durch Variation der Sensorkomponenten, der Testgase und der Messtemperatur konnten die Einflüsse der verschiedenen Parameter auf das Sensorsignal untersucht werden. So konnte gezeigt werden, dass der Einfluss der sensitiven $\mathrm{Cr}_{2} \mathrm{O}_{3}$-Schicht sehr groß ist. Mit steigender $\mathrm{Cr}_{2} \mathrm{O}_{3}$-Schichtdicke steigt auch die Spannungsdifferenz, gleichzeitig aber auch die Ansprechzeit des Sensors. Auch die Dicke der Zeolithschicht beeinflusst das Sensorsignal; bei Schichtdicken von $\sim 50 \mu \mathrm{m}$ wurde die größte Sensorantwort erhalten. Durch Zudosierung von Kohlenwasserstoffen wurde eine Spannungsabnahme, durch Zudosierung von Wasserstoff eine Spannungszunahme hervorgerufen. Der Pt-Gehalt in der Zeolithschicht hat einen gewissen Einfluss, allerdings ist nur bei Beaufschlagung mit Alkanen eine Abnahme des Sensorsignals mit steigendem Pt-Gehalt zu erkennen. Bei Messungen mit $\mathrm{H}_{2}$ wurden bei den Sensoren mit Pt-Gehalt von 2 Gew.-\% die größten Spannungsänderungen detektiert. Die Temperatur hatte bei den durchgeführten Messungen einen vergleichsweise geringen Einfluss auf das Sensorsignal. Bei $400{ }^{\circ} \mathrm{C}$ war allerdings eine Abnahme des Signals zu beobachten.

\section{Literatur}

[1] G. Hagen, R. Moos, Potentiometrische Gassensoren auf Zeolith-Basis, 9. Dresdner Sensor-Symposium, 7.-9. Dezember 2009, Dresden, Deutschland.

[2] G. Hagen, R. Moos, Planar Zeolite-Based Potentiometric Gas Sensors, Sensor Letters 9 (2011) 110-113.

[3] L. Smart, E. Moore, Einführung in die Festkörperchemie, Vieweg Braunschweig/Wiesbaden, S. 215-246, 1997.

[4] G. Hagen, A. Dubbe, F. Rettig, A. Jerger, Th. Birkhofer, R. Müller, C. Plog, R. Moos, Selective impedance based gas sensors for hydrocarbons using ZSM-5 zeolite films with chromium(III)oxide interface, Sensors and Actuators B 119 (2006) 441-448.

[5] G. Hagen, A. Schulz, M. Knörr, R. Moos, Four-Wire Impedance Spectroscopy on Planar Zeolite/Chromium Oxide Based Hydrocrabon Gas Sensors, Sensors 7 (2007) 2681-2692.

[6] S. Reiß, G. Hagen, R. Moos, Zeolite-based Impedimetric Gas Sensor Device in Low-cost Technology for Hydrocarbon Gas Detection, Sensors 8 (2008) 7904-7916.

[7] A. Tamási, K. Niesz, I. Pálinkó, L. Guczi, I. Kiricsi, Modifying the acidic properties of PtZSM-5 and PtY zeolites by appropriately varying reduction methods, in: R. Aiello, G. Giordano, F. Testa (Hrsg.), Studies in Surface Science and Catalysis, Band 142, Elsevier Science, S. 1801-1808, 2002. 